\title{
Half-night polysomnography: how is it compared to full-night polysomnography?
}

\author{
K.F. Chung
}

\section{To the Editor:}

I read with interest the paper by FANFulla et al. [1] on half-night polysomnography (HN-PSG). The authors found that there was a significant difference between apnoeahypopnoea indices (AHIs) recorded in the first and second portion of a standardized full-night polysomnography (FN-PSG). The statistical difference was more significant in patients who did not have rapid eye movement (REM) sleep during the first half of the night. The authors concluded that HN-PSG was not appropriate for evaluating patients with sleep-disordered breathing. In considering whether HN-PSG may be used to diagnose and assess the severity of sleep apnoea, the level of statistical difference between indices measured using HN-PSG and FN-PSG is less relevant than the magnitude of the difference. BLAND and Altman [2] described a graphical technique to compare the difference between readings by two methods to the mean of those two readings. The mean difference indicates the bias and the standard deviation of the differences indicates the error between the two methods. I used the Bland-Altman plot to examine the agreement between HN-PSG and FN-PSG in 37 consecutive patients who were diagnosed with obstructive sleep apnoea syndrome (OSAS) according to the International Classification of Sleep Disorders [3] and had a AHI $>10$ events $\cdot h-1$.

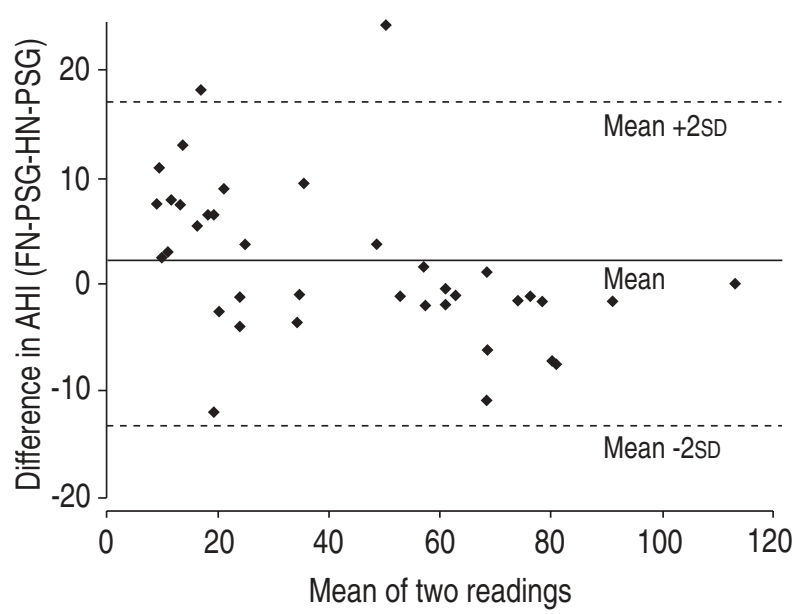

Fig. 1. - A Bland-Altman plot showing difference in apnoea-hypopnoea index (AHI) recorded during half-night $(\mathrm{HN})$ and full-night $(\mathrm{FN})$ polysomnography (PSG) against mean of indices. Mean (- ) difference in AHI $=$ two events $\cdot \mathrm{h}^{-1}$ and SD (-----) of the difference $=7.5$ events $\cdot h^{-1}$.
The 37 subjects were predominately male (male:female $35: 2)$ and $42.1 \pm 10$ yrs of age (mean \pm SD). The mean body mass index was 28.8 \pm 6.3 . During HN-PSG and FN-PSG, the mean total sleep times were $179 \pm 43$ and $412 \pm 54$ min, respectively. Ten subjects $(27 \%)$ had no REM sleep in the first half of the night. During HN-PSG and FN-PSG, the AHI (mean \pm SD) were $42.3 \pm 30.6$ and $44.4 \pm 27.1$, respectively, the number of $\breve{S} 4 \%$ oxygen desaturation per hour were $16.4 \pm 20.4$ and $17.6 \pm 18.3$, respectively, and the arousal index (mean $\pm \mathrm{SD})$ scored according to the American

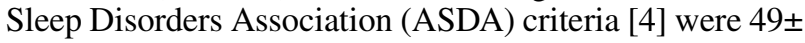
26.3 and $49.2 \pm 24.2$, respectively. There was no significant difference between the parameters measured during $\mathrm{HN}$ PSG and FN-PSG. The Bland-Altman plot of the AHIs recorded during HN-PSG and FN-PSG were shown in figure 1. The mean and SD of the differences in AHI using HN-PSG and FN-PSG were 2 and 7.5 events. $\mathrm{h}^{-1}$, respectively. The limits of agreement (mean $\pm 2 \mathrm{sD})$ and $95 \%$ confidence interval for the mean were -13 to +17 and -0.5 to +4 events. $\mathrm{h}^{-1}$, respectively. The difference between AHIs measured during HN-PSG and FN-PSG was $<10$ events $\cdot h^{-1}$ for most patients with mean AHI $>30$ events $\cdot h^{-1}$. The magnitude of the difference was less clinically significant than that in subjects with mean AHI $<30$ events $\cdot h^{-1}$.

I repeated the analysis in patients with mean AHI between 10 and 30 events $\cdot \mathrm{h}^{-1}(\mathrm{n}=17)$. The results were: mean $=4.8 ; \mathrm{SD}=7.1 ;$ limits of agreement $=-9.4-19$; and $95 \%$ confidence interval $=1.1-8.5$ events $\cdot h^{-1}$. HN-PSG considerably underestimated the severity of sleep apnoea and the AHI recorded during HN-PSG notably differed from that by a standardized full-night study. The results demonstrated that although there was no significant difference between the indices recorded during HN-PSG and FN-PSG, the actual magnitude of the difference could only be revealed by the Bland-Altman plot

I agree with FANFULLA et al. [1] that HN-PSG has a limited value for evaluating patients with sleep-disordered breathing. The assessment of the severity of sleep apnoea using HN-PSG in patients with mild OSAS is far from accurate.

\section{References}

1. Fanfulla F, Patruno V, Bruschi C, Rampulla C. Obstructive sleep apnoea syndrome: is the half-night polysomnography an adequate method for evaluating sleep profile and respiratory events? Eur Respir J 1997; 10: 1725 1729.

2. Bland JM, Altman DG. Statistical methods for assessing agreement between two methods of clinical measurement. 
Lancet 1986; i: 307-310.

3. Diagnostic Classification Steering Committee; Thorpy MJ, Chairman. International Classification of Sleep Disorders: Diagnostic and Coding Manual. Rochester, MN, American Sleep Disorders Association, 1990.
4. Altas Task Force. EEG arousals: scoring rules and examples. Sleep 1992; 15: 174-184.

\section{K.F. Chung}

Dept of Psychiatry, University of Hong Kong, Pokfulam, Hong Kong. Fax: 85228551345.

\section{REPLY}

The increasing requests of a full-night study represent a great problem for several sleep laboratories, and consequently it is necessary to better define the most adequate and less expensive procedure to obtain a diagnosis of sleepapnoea syndrome. Half-night polysomnography (PSG) was proposed for achieving this objective without losing the information given by 16-channels polysomnography. Before the split night method, nocturnal pulse-oximetry or transcutaneous assessment of blood gases was the only extensive screening method for evaluation of sleep-disordered breathing.

The data reported by K.F. Chung obtained from a large population are very similar to those we described and confirm the inadequacy of full-night polysomnography, in the assessment of obstructive sleep apnoea (OSA). Furthermore, in the last American Lung Association (ALA)/American Thoracic Society (ATS) meeting (Chicago, 1998), PARACHA et al. [1] found that split-night PSG underdiagnosed the presence of OSA and underestimated the OSA severity particularly in mild and moderate cases.

A Bland-Altman plot may graphically better represent the differences between apnoea-hypopnoea index (AHI) calculated in the full- and the split-night recording. However, Altman and Bland's methodology is particularly desig-ned for comparing two different measurement techniques. In a validation study of the adequacy of split-night in diagnosing OSA syndrome, the problem is the accuracy of split-night in predicting the value of AHI recorded in the full-night registration. For this reason, we presented in the paper a linear prediction model including as a critical co-efficient the presence or absence of rapid eye movement phase sleep in the first part of the night. Our results are very similar to those reported by K.F. Chung, although the statistical approach was different.

We conclude that a further effort is needed from scientific organizations to define a new plan for evaluating patients suffering from sleep-disordered breathing including, as a first-line assessment, a clinical approach with new clinical questionnaires as well as home eight-channel polysomnography; thus, full-night polysomnography could be performed only in patients with a severe alteration or with associated diseases or in patients with meaningful alterations.

\section{Reference}

1. Paracha MI, Dasqupta A, Golish J, et al. Split night polysomnogram underdiagnosis the presence and severity of obstructive sleep apnea. Am J Respir Crit Care Med 1998; 157: A650.

\section{F. Fanfulla}

Fondazione S. Maugeri, Centro Medico di Montescano, 27040 Montescano (PV), Italy. Fax: 3938561386. 